\title{
Meningism following Salmonella virchow food poisoning
}

\author{
P.G. Norris \\ Department of Medicine, Chelmsford \& Essex Hospital, London Road, Chelmsford, UK.
}

\begin{abstract}
Summary: Thirty six patients were admitted to hospital as a result of Salmonella virchow infection during an outbreak of food poisoning in Essex in 1984. Out of 12 patients with evidence of bloodstream invasion, one third presented primarily with meningism and attention is drawn to this unusual clinical picture.
\end{abstract}

\section{Introduction}

Salmonella virchow has become increasingly recognized as a cause of food poisoning in England and Wales-during the last 4 years it has been the second or third most commonly reported salmonella serotype to be identified in human faeces (PHLS Communicable Diseases Surveillance Centre, unpublished data). Although it was originally isolated (Kaufman, 1930) from a patient with a typhoid-like illness, the propensity of $S$. virchow for bloodstream invasion was not noted until 1972 (Mani et al., 1974). Experience of a larger outbreak in Essex in 1984 is reported and attention drawn to 4 patients with an invasive illness who presented with marked meningeal irritation.

\section{Outbreak}

Over a 3 month period from May to July 1984, positive stool and/or blood cultures for S. virchow were obtained from 297 patients during an outbreak of food poisoning in Mid-Essex. The medical records of the 36 patients admitted to hospital as a result of their infection were later examined.

Twenty three patients were admitted with gastroenteritis, the duration of symptoms before admission varying between 1 and 28 days (mean 6.4 days). Blood cultures were clinically indicated in 14 patients and $S$. virchow isolated from 11. Eight of the latter presented primarily with a systemic illness characterized by a high spiking fever and marked systemic upset. All patients in this group also suffered from diarrhoea, severe enough to cause electrolyte depletion in 3 cases, and all, apart from one case in which a specimen was not obtained, had positive stool cultures. Four other

Correspondence and present address: P.G. Norris, M.A., M.R.C.P., St. John's Hospital for Diseases of the Skin, Homerton Grove, London E9 6BX, UK

Accepted: 12 February 1986 patients with evidence of bloodstream invasion presented primarily with neck stiffness; their case histories are described below.

\section{Case histories}

\section{Case 1}

A previously well 20 year old student presented with a 7-day history of increasing headaches and anorexia, followed by rigors and sweating. Two days before admission he developed photophobia. There was no history of diarrhoea. On examination he was pyrexial $\left(39^{\circ} \mathrm{C}\right)$ and had marked neck stiffness. Biochemical and microbiological examination of cerebrospinal fluid (CSF) was normal. Blood cultures and a rectal swab on admission both grew $S$. virchow.

Treatment with oral cotrimoxazole was commenced when the result of the blood culture was received. Defervescence was evident within 36 hours and he was discharged home a week later.

\section{Case 2}

A 34 year old man was admitted with a 5-day history of severe frontal headaches, muscle pains, sweats and photophobia. There were no gastrointestinal symptoms. He was pyrexial $\left(39.2^{\circ} \mathrm{C}\right)$, had mild neck stiffness and Kernig's sign was positive. A rectal swab and blood cultures grew $S$. virchow. Lumbar puncture was not performed. After commencing treatment with intravenous chloramphenicol his fever settled rapidly and he returned home 10 days later.

\section{Case 3}

A 20 month old boy presented as an emergency with a 24-hour history of drowsiness and vomiting, but no

(C) The Fellowship of Postgraduate Medicine, 1986 
diarrhoea. The only positive clinical findings were a pyrexia of $38.5^{\circ} \mathrm{C}$ and marked neck stiffness. CSF was biochemically and microbiologically normal. A rectal swab grew $S$. virchow although blood cultures drawn at the time of admission were sterile. He was treated empirically with intravenous ampicillin and flucloxacillin and made a full recovery.

\section{Case 4}

A 21 year old man was admitted with a 4-day history of headache and photophobia, and a 24-hour history of lower abdominal pain with mild diarrhoea and one episode of vomiting. Clinical examination revealed a pyrexia of $39.0^{\circ} \mathrm{C}$, right sided abdominal tenderness, and neck stiffness. No white blood cells were seen on microscopy of the CSF which was sterile. The blood and stool cultures both grew S. virchow. Cotrimoxazole was commenced on receipt of the blood culture report and his pyrexia subsided over the next 48 hours.

\section{Discussion}

Out of 297 individuals with proven Salmonella virchow infection, blood cultures were positive in 11 of 14 samples and only one other of the patients admitted to hospital (case 3) had clinical evidence of septicaemia. It is possible that a small number of the cases not requiring hospital admission had an undetected septicaemia, but this would still appear to represent a markedly lower incidence of bloodstream invasion than in a comparable multi-source but circumscribed outbreak of $S$. virchow food poisoning described by Mani et al. (1974) in which 7 out of a total of 21 cases had a septicaemic illness.

It is of interest that 4 of the patients with an invasive illness presented specifically with neck stiffness, in 3 cases leading to lumbar puncture. The mean age of the 4 patients with meningism was 19.2 years compared with an average of 61.6 years for the other 8 patients with positive blood cultures.

Positive stool cultures were obtained in all four cases with meningeal irritation; gastrointestinal symptoms were however absent in 3 of these patients and a late and mild feature in case 4 . In general, diarrhoea is uncommon in invasive salmonellosis (Saphra \& Winter, 1957; Christie, 1980) - this was not, however, the experience in this outbreak where all 8 patients presenting with a non-specific systemic illness suffered diarrhoea. $S$. virchow may be atypical in this respect as previous reports of septicaemic patients have noted frequent or loose stools in over two thirds of cases (Mani et al., 1974; Todd \& McMurdoch, 1983).

Neurological abnormalities, including confusional states, convulsions, parkinsonism and polyneuropathy, occur in up to $50 \%$ of patients with typhoid fever (Koshla et al., 1977), but meningitis is rare (Chanmugam et al., 1978). Meningitis is also an uncommon but recognized focal manifestation of other salmonella serotypes capable of invading the blood stream (Saphra \& Winter, 1957). However, meningeal irritation has not hitherto been emphasized as a possible presentation of salmonellosis and only one case following $S$. virchow infection in an 18 year old patient has been documented (Todd \& McCMurdoch, 1983). From this outbreak, it is clear that in younger patients bloodstream invasion by $S$. virchow not uncommonly results in meningism, usually with no gastrointestinal symptoms, and this should be remembered as a potentially misleading clinical picture.

\section{Acknowledgements}

I am grateful to the physicians and microbiology departments of the following hospitals for their kind assistance: Basildon Hospital, Chelmsford \& Essex Hospital, Harold Wood Hospital, Romford; Rush Green Hospital, Romford; Southend Hospital, St Mary's Hospital, Colchester.

\section{References}

CHANMUGAM, D., MACHADO, V. \& MININDUKULASURIYA, J.L. (1978). Primary Salmonella typhi meningitis in an adult. British Medical Journal, 1, 152.

CHRISTIE, A.B. (1980). In Infectious Diseases: Epidemiology and Clinical Practice. p. 31. Churchill Livingstone: Edinburgh, London, Melbourne, New York.

KAUFMAN, F. (1930). Neue Serologische Typen der Paratyphusgruppe. Zeitschrift für die gesamte Hygiene und ihre Grenzgebiete, 111, 221.

KHOSHLA, S.N., SRIVASTAVA, S.C. \& GUPTA, S. (1977). Neuropsychiatric manifestations of typhoid fever. Journal of Tropical Medicine and Hygiene, 80, 95.

MANI, V., BRENNAND, J. \& MANDAL, B.K. (1974). Invasive illness with Salmonella virchow infection. British Medical Journal, 2, 143.

SAPHRA, L. \& WINTER, J.W. (1957). Clinical manifestations of salmonellosis in man. New England Journal of Medicine, 256, 1128.

TODD, W.T.A. \& McMURDOCH, J. (1983). Salmonella virchow: a cause of significant bloodstream invasion. Scottish Medical Journal, 28, 176. 